\title{
POURQUOI LE MASCULIN À VALEUR GÉNÉRIQUE EST-IL SI TENACE, EN FRANÇAIS?
}

\author{
Daniel Elmiger
}

\begin{abstract}
This article attempts to establish a connection between linguistics and psychoanalysis, using as an example the genericity of masculine forms, a topic which is central, in French, in the debate about the "féminisation des textes" (i. e. avoiding generically used masculine forms) and which seems to be collectively repressed as opposed to the "féminisaton du lexique" (i. e. the coining of female-specific nouns. Moreover, the conceptualisation of the «creation » of female-specific nouns (by means of an inflexional or derivational process) is discussed and exemplified by analogy with the two narrations of the creation of the first humans in Genesis (chapter 1).
\end{abstract}

Keywords: lexical feminisation; nonsexist language; generic masculine; flexion; derivation; Bible; psychanalysis.

Résumé : Cet article essaie de faire un lien entre la linguistique et la psychanalyse, à propos de la question de la généricité du masculin, qui se trouve au centre de la féminisation des textes et qui semble être «refoulée » collectivement par rapport à la féminisation lexicale. En outre, la question de la conceptualisation de la «formation » des noms féminins (est-ce un procédé de flexion ou de dérivation ?) est abordée et exemplifiée, par analogie, avec les deux récits de la création de l'être humain dans la Genèse (chapitre 1).

Mot-clés : féminisation du lexique ; féminisation des textes ; masculin générique ; flexion ; dérivation ; Bible ; psychanalyse.

\section{Linguistique et psychanalyse}

Dans les études qui la constituent, la linguistique doit s'en tenir pour l'essentiel aux énoncés du sujet parlant ainsi que, notamment en sociolinguistique, aux conditions externes dans lesquelles évoluent les sujets parlants et leur communication. Le monde interne des sujets parlants - leurs pensées, leurs motivations conscientes et inconscientes reste pour l'essentiel inaccessible, du moins pour ce qui passe à travers les filtres de la parole, qui structurent, sélectionnent et réorganisent un vécu intérieur qui ne semble être que partiellement langagier.

Ainsi, l'étude du monde interne, champ d'études de la psychologie et notamment de la psychanalyse, n'entre que très marginalement dans les études linguistiques, ce qui en limite - nécessairement, mais aussi malheureusement - leur pertinence. ${ }^{1}$ Pourtant, le

\footnotetext{
1 Il est d'autant plus intéressant si certain-e-s parviennent à puiser dans les deux champs, comme c'est le cas d'Anne-Marie Houdebine - à qui cet article est dédié -, qui est, entre autres, sociolinguiste et psychanalyste.
} 
monde préconscient ou inconscient semble largement coresponsable de ce qui contribue à conditionner nos actes (langagiers ou non langagiers) : les motivations, représentations, inhibitions, interdits, etc.

La théorie linguistique gagnerait certainement à entrer en dialogue avec la psychologie et la psychanalyse ; dans le cadre du présent article, nous nous limiterons à un éclairage très partiel, simplifié et réducteur, qui ne saurait prétendre à une validité autre qu'anecdotique.

\section{Régulation des conflits face aux normes linguistiques}

La posture du sujet face aux normes de son environnement - ainsi que les conflits qui en découlent - sont au centre de l'intérêt tant de la linguistique que de la psychanalyse. Les travaux d'Anne-Marie Houdebine ont permis d'affiner les diverses normes langagières et de décrire les différentes conceptions de norme (tantôt dans le sens de "régularité, ordre habituel» et tantôt dans le sens de " prescription, règle à suivre $»^{2}$ Ainsi, elle distingue entre normes objectives (ce qui est normal) vs normes subjectives (ce qui est normatif), par rapport auxquelles le sujet parlant doit se positionner. Cela ne s'observe pas directement; Houdebine définit l'imaginaire linguistique comme « le rapport du sujet à la lalangue (Lacan) et à La Langue (Saussure) » qui est « repérable par ses commentaires évaluatifs sur les usages ou les langues » (Moreau 1997).

La théorie freudienne décrit, de manière générale, les liens (et les tensions) entre les différentes instances de l'appareil psychique (le moi, le surmoi et le ça) et le monde externe, la "réalité ». ${ }^{3}$ Comment situer ces trois instances par rapport aux normes langagières telles qu'elles apparaissent dans le modèle de l'imaginaire linguistique?

Pour l'individu subjectif, les normes objectives (qui font l'objet de la description objective, souvent en porte-à-faux avec les idées reçues ou l'intuition des chercheur-e-s) ne semblent jouer qu'un rôle marginal : pour de nombreuses personnes, la nécessité d'une linguistique descriptive, neutre, semble moins pertinente que celle d'une linguistique prescriptive.

En revanche, les normes subjectives (notamment les normes évaluatives, communicationnelles, fictives, prescriptives) sont certainement celles qui sont le plus présentes auprès des sujets parlants. ${ }^{4}$

\section{Un exemple : dissonance cognitive en matière de féminisation de la langue}

Dans les pays et régions francophones, la remise en question féministe du langage traditionnel, souvent jugé sexiste ou androcentriste, a surtout porté sur le vocabulaire, où de nombreux termes désignant des métiers, fonctions, grades ou titres, qui n'avaient qu'une forme masculine, ont été « féminisés ». Ceci a donné lieu à un certain nombre

2 Cf. Houdebine (1998).

3 Il va de soi que la réalité n'est pas immédiatement perceptible pour les sujets et fait l'objet d'une construction personnelle, continuellement réadaptée et ajustée à l'entourage.

4 Les différentes instances de la personnalité auraient-elle des rapports différenciés aux normes? On pourrait postuler que le moi entretient un rapport d'adulte avec les normes, en visant l'observation des normes tout en cherchant à maintenir son autonomie. Le surmoi peut certainement être mis en relation avec les normes prescriptives (étayées par des discours institutionnels), qui délient le sujet parlant individuel de sa responsabilité (mais aussi partiellement de sa liberté de parole). Enfin, on peut postuler que la posture du ça est celle d'un refus de toute norme (prescriptive) perceptible en tant que telle, avec des désirs de transgression (contrebalancés par la crainte de non-conformité ou de rejet). 
de « guides » d'aide à la féminisation (sous forme de listes de mots ou de règles de formation du féminin) ou de dispositions légales ayant pour but d'activement favoriser un vocabulaire «féminisé ». Si cela semble important et utile (du fait que le système des noms communs de personnes français repose sur plusieurs sous-systèmes partiellement contradictoires), il est tout de même possible de faire deux constats : d'un côté, cette " féminisation du lexique » portait surtout sur le lexique désignant des fonctions socialement valorisées (les métiers, professions, titres, etc.) et non pas sur l'ensemble des noms communs de personne ${ }^{5}$, et de l'autre, la question de la gestion des formes génériques a souvent été esquivée, notamment en France. ${ }^{6}$

Ainsi, la question de la « féminisation de la langue » a donné lieu à deux conflits : celui de l'intégration de nouvelles formes dans un système morphologique irrégulier (gestion de la néologie) et celui de la référence équitable des sexes par des moyens langagiers (formes génériques ou formes alternatives).

\section{La difficile gestion de la néologie}

Les causes qui ont donné naissance (notamment durant le $X X^{\mathrm{e}}$ siècle) à de nombreuses formes féminines nouvelles sont nombreuses et reliées entre elles : raisons politiques et sociales (accès des femmes à de nombreuses fonctions et métiers), revendications féministes (parfois relayées par des actions politiques), évolution des usages, etc.

Une manière active de résoudre le conflit lié à la néologie consiste à distinguer entre la « bonne » et la « mauvaise » féminisation. Les jugements s'appuient sur diverses normes prescriptives et subjectives, p. ex. :

- critères de régularité : formes régulières (c'est-à-dire qui sont formées selon des modèles morphologiques existants, p. ex. chercheuse) vs formes irrégulières (p. ex. chercheure) ; 7

- critères esthétiques : formes qui plaisent vs formes qui ne plaisent pas (qui «font mal » aux yeux ou aux oreilles) $i^{8}$

- critères fonctionnels : formes désignant une personne (et permettant ainsi une féminisation) vs formes désignant une fonction ; 9

- critères de légitimité : formes légitimées (par les instances de norme prescriptive) vs formes non légitimées (mais utilisées par les personnes désignées, p. ex. la cheffe).

Ceci a pour conséquence que la question de la féminisation lexicale continue d'être dans la nécessité de se légitimer, tant au niveau individuel (des formes individuelles) qu'au niveau collectif (l'utilisation des formes féminines). Quel que soit le système de légitimité, les sujets parlants doivent ainsi choisir les bonnes formes (si possibles légitimées par les instances prescriptives et l'usage), ce qui trahit une vision docile envers le langage,

\footnotetext{
Cela semble être vrai surtout pour les «guides de féminisation » (cf. Elmiger 2000) ; certains textes à visée plus féministe (p. ex. Larivière (2000a et 2000b) abordent le champ des désignations humaines de manière plus globale.

6 Cela est moins le cas dans les publications canadiennes, influencées par la discussion en anglais (cf. p. ex. le guide récent de l'Office québécois de la langue française, 2007), ainsi que d'autres guides (p. ex. Moreau 2001).

7 Cf. la place récurrente de certaines formes inhabituelles (p. ex. recrue, estafette, vigie) et de certaines formes difficiles à féminiser (médecin, successeur) dans l'argumentation contre la féminisation lexicale.

8 Cf. Elmiger (2008: 2003 ss.).

9 Cf. à ce propos l'argumenation du rapport de la Commission générale de terminologie et de néologie (1998).
} 
où ce n'est pas le sujet parlant qui se sert librement des matériaux linguistiques dont il a besoin ; toutefois, ce faisant il reste en position de servilité par rapport à « la langue ${ }^{10}$

\section{Les formes féminines : complémentaires ou dérivées par rapport aux formes masculines?}

Nous avons montré ailleurs (Elmiger 2008) quelle influence la conceptualisation du rapport entre noms communs de personne féminins et masculins avait sur la légitimité de la " féminisation » (qui, elle, implique nécessairement une agentivité et une postériorité des formes féminines) : le système des noms communs de personne est-il considéré comme faisant partie de la flexion ou comme étant un cas de dérivation? En cas de flexion, la question de la nécessité (ou de la permission) de féminiser ne se pose pas, car l'utilisation d'une forme masculine (elle...le président), en parlant d'une femme, serait tout aussi fausse que l'utilisation d'un adjectif masculin (elle...intelligent). En cas de dérivation en revanche, il s'agit de décider au cas par cas si le processus dérivationnel peut être appliqué - et si l'antériorité de la "forme de base » (en général la forme masculine) par rapport à la forme dérivée (en général féminine) est clairement statuée (pour une discussion des arguments, cf. Elmiger 2008 : 72-77).

\section{Une digression: les deux récits de la création de l'être humain dans la Genèse $e^{11}$}

Les deux conceptualisations du rapport entre formes féminines et formes masculines se trouvent, mutatis mutandis, dans la Bible ; la première mention de la création de l'être humain se trouve au premier chapitre de la Genèse :

Genèse $1.277^{12}$

Dieu créa l'homme à son image, à l'image de Dieu il le créa ; mâle et femelle il les créa.

Cette brève description ne présuppose pas d'antériorité d'un sexe par rapport à l'autre ; les deux étant une instanciation de l'être humain (traduit en français comme " homme »

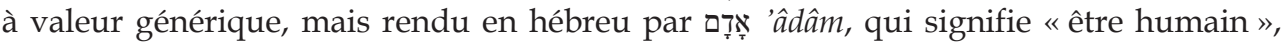
mais aussi «l'humanité » et, en tant que nom propre, "Adam »). ${ }^{13}$ Même si le terme utilisé pour désigner l'homme (= être humain) est aussi le nom du premier homme, il est tout de même spécifié d'emblée que l'être humain a été créé sous les deux formes " mâle et femelle », sans que l'une soit antérieure à l'autre (à noter que les deux sont ainsi créés "à l'image de Dieu »). ${ }^{14}$

L'autre récit de la création de l'être humain se trouve au chapitre 2. Il est un peu plus long que le premier et ajoute au thème de la création de l'être humain celui de l'émancipation des humains par rapport à la souveraineté divine. Il commence par la création de l'homme ('âdâm), à qui le Seigneur donne d'abord la vie et ensuite une première injonction, c'est-à-dire celle de ne pas manger de l'arbre de la connaissance. La compagnie

${ }^{10}$ Cf. à ce propos les nombreuses formules thématisant le « respect » que les locuteurs devraient témoigner envers la langue : respecter le français / la langue / l'orthographe, etc.

${ }^{11}$ Je remercie René Péter-Contesse pour son aide précieuse lors de la rédaction de ce chapitre.

12 Traduction œcuménique de la Bible. 2010. Paris : Cerf.

13 Quant à mâle et femelle, ils correspondent à זִ ְ̦ (zâkâr et neqébâ) qui s'utilisent à la fois comme adjectifs et comme noms communs de personne.

${ }_{14} \mathrm{D}^{\prime}$ autres interprétations de ce passage ont été faites, p. ex. en proposant l'hypothèse d'un être humain intersexuel (Gross 1999). 
que Dieu prévoit d'abord pour l'homme n'est pas la femme mais les animaux : dans ce deuxième récit de la création, l'homme (mâle) est ainsi confronté à la norme, mais il lui est également accordé le pouvoir de nommer le monde à sa guise en prenant possession, symboliquement (par son premier acte de langage), des animaux autour de lui.

Ce n'est que dans un deuxième temps (lorsqu'il s'avère que les animaux ne sont pas l'aide qu'il faut à l'homme-mâle) que Dieu crée la femme en la formant à partir de (la côte de) l'homme : donc par dérivation.

Genèse 2.7-23

7 Le SEIGNEUR Dieu modela l'homme avec de la poussière prise du sol. [...]

16-23 Le SEIGneur Dieu prescrivit à l'homme : «Tu pourras manger de tout arbre du jardin, / mais tu ne mangeras pas de l'arbre de la connaissance de ce qui est bon ou mauvais car, du jour où tu en mangeras, tu devras mourir. » Le SEIGNEUR Dieu dit : " Il n'est pas bon pour l'homme d'être seul. Je veux lui faire une aide qui lui soit accordée. » Le SEIGNEUR Dieu modela du sol toute bête des champs et tout oiseau du ciel qu'il amena à l'homme pour voir comment il les désignerait. Tout ce que désigna l'homme avait pour nom " être vivant "; l'homme désigna par leur nom tout bétail, tout oiseau du ciel et toute bête des champs, mais pour lui-même, l'homme ne trouva pas l'aide qui lui soit accordée. Le SEIGNEUR Dieu fit tomber dans une torpeur l'homme qui s'endormit; il prit l'une de ses côtes et referma les chairs à sa place. Le SEIGNEUR Dieu transforma la côte qu'il avait prise à l'homme en une femme qu'il lui amena. L'homme s'écria : "Voici cette fois l'os de mes os et la chair de ma chair, celle-ci, on l'appellera femme car c'est de l'homme qu'elle a été prise. »

Ce deuxième récit établit ainsi une claire succession dans l'apparition des deux sexes, décrivant la femme (ainsi que son appellation ה

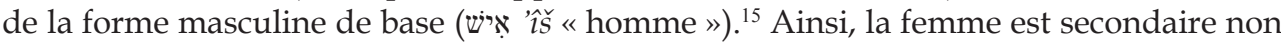
seulement du point de vue physique, mais aussi par rapport à l'instauration du langage et des normes.

\section{Refoulement}

En français, la conception des genres (ainsi que celle des formes portant des marques de genre) se fait le plus souvent selon ce deuxième schéma : le masculin est en général considéré comme le genre premier, supposé neutre et non marqué, qui peut englober le féminin, envisagé comme secondaire, dérivé et marqué. ${ }^{16}$ Cette conception permet entre autres de maintenir le statut générique du genre masculin.

À notre avis, la focalisation sur la féminisation lexicale permet de ne pas entrer en matière sur le véritable enjeu que présentent les noms communs de personne masculins et féminins : la féminisation discursive. En termes psychologiques, cela équivaut d'une part à une fixation (sur la casuistique lexicale) et, d'autre part, à un processus de refoulement qui est une manière passive de résoudre le conflit généré par la féminisation de la langue. Plusieurs indices permettent d'étayer cette thèse. Premièrement, comme

15 En tchèque, les traductions de la Bible se sont servies de différents procédés pour rendre ce procédé dérivatif: par exemple, dans la version de 1613 de la Bible Kralická, il est question de muž (" être humain mâle " et de mužatka («femme hommasse »), tandis que dans une traduction récente (Bible, překlad 21. stoleti), les termes moins négativement connotés manžel/manželka («époux/épouse ») ont été retenus.

${ }^{16}$ Labrosse (1996 : 28-31) énumère plusieurs qualificatifs qui ont été donnés au genre masculin (genre «premier », «plus fort », «plus noble », « le plus digne ou le plus louable »). Aujourd'hui, on dit communément que c'est le genre «qui l'emporte». 
nous l'avons écrit plus haut, la question de l'écriture non discriminatoire (la gestion/ l'évitement des formes (masculines) à valeur générique ; l'utilisation de formes alternatives, etc.) est quasi-absente des discours féministe, politique, médiatique et individuel. En outre, les guides d'aide à l'écriture non discriminatoire sont très rares dans l'espace francophone (en particulier en France) et ne visent pas, en général, le bannissement des formes masculines à valeur générique.

Ensuite, les résultats de la recherche psycholinguistique (qui montrent clairement que l'utilisation du seul masculin à valeur générique suscite des représentations majoritairement masculines) sont moins exploités dans le discours féministe que dans d'autres pays. Finalement, le langage des voix féministes est pour l'essentiel traditionnel en ce qui concerne la gestion de la généricité. Les formes alternatives (doublons intégraux ou formes abrégées) n'ont que très peu de visibilité dans l'espace public.

\section{Conclusion : une dénégation collective?}

Pourquoi l'inégalité symbolique, véhiculée par le masculin à valeur générique, est-elle si peu contestée dans l'espace francophone, en comparaison avec d'autres langues? Toutes sortes d'arguments sont avancées, de type normatif (« le masculin l'emporte » ou «L'Académie s'y oppose »), pragmatique (« c'est trop compliqué »), stylistique («c'est nuisible à la clarté et à l'élégance » ou essentialiste (« Le génie de la langue ne le permet pas »). Il est indéniable que l'abandon (partiel ou complet) du masculin à valeur générique n'est pas simple à mettre en pratique. Néanmoins, on peut se demander si la nonthématisation de la question ne relève pas d'un évitement collectif du conflit et d'une dénégation d'une inégalité symbolique crasse.

L'expérience dans d'autres langues (comme l'anglais et l'allemand) montre que les résistances par rapport à une remise en question des valeurs sémantiques des genres masculin et féminin se sont avérées certes considérables, mais que suite à une mobilisation à divers niveaux (surtout féministe et politique), les représentations et les pratiques ont effectivement changé : dans les deux langues, la perception des genres n'est plus la même qu'il y a 40 ans et, dans certains textes particulièrement sensibles, l'usage du seul masculin à valeur générique ne semble plus aller de soi (cf. Elmiger 2012 et 2013). Une gestion consciente de la conflictualité semble ainsi possible malgré toutes les difficultés et les résistances actives ou passives. Il sera intéressant de voir si, en français, la manière passive de gérer le conflit lié à la « féminisation des textes », qui semble être l'expression d'un évitement de conflit, évoluera à l'avenir et par quelles voies les normes prescriptives seront remises en question.

\section{Bibliographie}

Durrer, Sylvie - Jufer, Nicole - PAHud, Stéphanie (2009), La place des femmes et des hommes dans la presse écrite généraliste de Suisse romande des années 80 à nos jours, Zürich : Seismo (collection Questions de genre).

ElMiger, Daniel (2000), « Les guides de féminisation allemands et français : La Suisse au carrefour d'influences différentes », Bulletin suisse de linguistique appliquée 72, 211-225.

Elmiger, Daniel (2008), La féminisation de la langue en français et en allemand. Querelle entre spécialistes et réception par le grand public. Paris: Honoré Champion. 
Elmiger, Daniel (2009), «Féminisation de par la loi : la nouvelle < loi sur les langues > suisse et la formulation non sexiste », LeGes : législation E évaluation 1, 57-70.

Elmiger, Daniel (2012), «Quelle évolution dans le domaine de la féminisation de la langue? Constitution d'un corpus pour la description diachronique et synchronique des usages ", in : BAIDER, Fabienne H. - Elmiger, Daniel, éds. Intersexion. Langues romanes, langues et genre, München : Lincom, 97-105.

Elmiger, Daniel (2013), « The government in contact with its citizens: Translations of federal information in multilingual Swiss administration », Gender E Language $7 / 1,59-74$.

Gross, Sally (1999), «Intersexuality and Scripture, Theology and Sexuality 11, 65-74.

Houdebine[-Gravaud], Anne-Marie (1998), « Insécurité linguistique, imaginaire linguistique et féminisation des noms de métiers ", in : SingY, Pascal, éd. : Les femmes et la langue. L'insécurité linguistique en question, Lausanne - Paris : Delachaux et Niestlé, 155-176.

Labrosse, Céline (1996), Pour une grammaire non sexiste, Montréal : Éditions du remue-ménage.

LaRIVIÈre, Louise-L. (2000a), Pourquoi en finir avec la féminisation linguistique ou à la recherche des mots perdus, Montréal : Boréal.

LARIVIÈRE, Louise (2000b), Comment en finir avec la féminisation linguistique ou Les mots pour LA dire. Paris : Editions 00h00.

Moreau, Marie-Louise, éd. (1997), Sociolinguistique. Concepts de base, Sprimont : Mardaga.

Moreau, Thérèse (2001), Écrire les genres. Guide romand d'aide à la rédaction administrative et législative épicène. Conférence latine des déléguées à l'égalité (éd.). Genève : État de Genève.

Office québéCoIs de la langue française (2007), Avoir bon genre à l'écrit. Guide de rédaction épicène, Québec: Office québécois de la langue française.

ReY, Alain (1972), "Usages, jugements et prescriptions linguistiques », Langue française 16, 4-28.

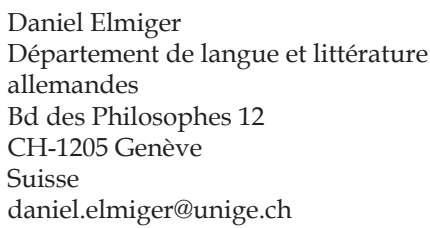

